IOS Press

\title{
A survey of hybridisation methods of GNSS and wireless LAN based positioning system
}

\author{
Philipp Richter*, Manuel Toledano-Ayala, Genaro M. Soto-Zarazúa and Edgar A. Rivas-Araiza \\ Universidad Autónoma de Querétaro, Cerro de las Campañas s/n., 76010 Santiago de Querétaro, Qro., México
}

\begin{abstract}
Self-localisation has become a matter of course in our daily life. The emerging market of mobile devices still boosts the demand for seamless, ubiquitous positioning. The repertoire of sensors serviceable for localisation provided by current mobile devices is large; however the main positioning systems used today are based on wireless local area networks (WLAN), cellular networks and certainly global navigation satellite systems (GNSS). Because of the accuracy, the vast deployment and the channel characteristics, researchers have been focused on WLAN based positioning systems (WPS) in particular, to achieve seamless positioning. This paper reviews the latest data fusion approaches to seamless positioning by GNSS and WPS. In accordance to the level of data fusion, several approaches are categorised and briefly presented, differences in performance of these approaches are highlighted and future challenges identified.
\end{abstract}

Keywords: Seamless positioning, GNSS, WLAN, sensor data fusion

\section{Introduction}

The exponentially emerging mobile device market has created a vast demand of location based services (LBS). For many of these applications a seamless indoor/outdoor, a really ubiquitous localisation system is crucial. Outdoors, the NAVSTAR GPS is filling that role since 20 years and many advances in the development GNSSs towards localisation in urban canyons and indoors have been made $[15,54,55]$. The sensitivity could be increased, including the development of specialised high-sensitivity GNSS (HS-GNSS) receiver; multiple constellations are available nowadays, increasing the coverage; multiple frequencies can be used to mitigate ionospheric influences, and several augmentation systems have been developed in order to improve accuracy and the time-to-first-fix (TTFF). However, a robust indoor localisation solely based on GNSS is still not possible [79]. Much less when referring to LBS, which rely on a large number of users needing affordable and portable devices. Hence, it is widely suggested to use additional sensor information

*Corresponding author. E-mail: philipp.richter@uaq.mx, Tel. +52 19212006023 , Fax: +52 19212006006 . from inertial measurement systems or wireless communication systems to overcome that problem $[15,18$, $38,62]$. For indoor localisation several technologies have been investigated $[25,38,62]$, from whose a localisation technology based on the IEEE 802.11 standard for implementing WLANs, seems to be the most promising approach $[13,55]$ providing a good tradeoff between accuracy, scalability, robustness, complexity, and costs. Moreover, WLAN positioning systems and GNSSs have complementary characteristics. A GNSS is dependent on line-of-sight conditions and works best in open areas. IEEE 802.11 signals penetrate objects and walls and especially the fingerprinting positioning technique, a pattern recognition method, benefits from an irregular signal strength distribution caused by signal shadowing, scattering and so on. The biggest advantage of using a localisation based on WLAN, is probably the already existing infrastructure and a transceiver chip in every modern mobile device. By combining these two positioning techniques rigorously, the coverage area and robustness is increased. Such a system can provide a seamless and ubiquitous localisation.

This work intends to summarise, categorise and compare existing data fusion techniques of GNSS and 
WPS. It does not focus on how sensory data is processed to derive directly a position, rather how data is fused to obtain a position. The paper is organised as follows. An overview of GNSS, how WLAN can be employed as positioning system, and of hybridisation schemes in general is given in Section 2. In Section 3 works are briefly presented, that fuse data of GNSS and WPS. The presentation of these approaches is arranged with respect to the level at which the data fusion is accomplished. In Section 4 the performance of the categories is assessed with respect to accuracy, precision, robustness, complexity and cost. A conclusion is drawn in Section 5 and an outlook can be found in Section 6.

\section{Background}

\subsection{GNSS}

The first satellite navigation system, called TRANSIT, was developed in the late fifties [9]. About fifteen years later NAVSTAR GPS and GLONASS were taken into service but it took until the mid nineties that both systems were fully operational [55].

GNSSs are designed as a positioning system providing an accurate, continuous three-dimensional position and velocity, and time information. Fundamental for all GNSSs is the unidirectional link between the satellites and the (passive) receiver, thus allowing an unlimited number of users. In GPS, Galileo and in future in GLONASS too [9], a code division multiple access (CDMA) scheme is used to identify each satellite. The main purpose of that CDMA scheme is to carry out the time measurement. The codes are designed to be orthogonal to each other, to create a clear autocorrelation peak and no cross-correlation peak. The time-of-arrival (TOA) of a signal is obtained by correlating in incoming code with a local replica of the same code. To find the starting point of the code, the local replica is shifted until the codes coincide and the correlation peaks. The TOA corresponds to the time difference that the local code had to be shifted. To estimate a position, additional to the TOA the positions of the transmitter must be known to the receiver. That information is send via the same signals within the so called navigation message. This message contains in addition to the satellite precise orbital information (ephemeris) the satellite clock correction data. TOAs of at least four satellites are converted into distances. Because the time measurements contain the error of the receiver clock, these distances are called pseudo ranges. Via lateration of four satellites the receiver clock error and a three-dimensional position is estimated.

To solve the lateration equation non-iterative (Bancroft's algorithm) and iterative algorithm (Kalman filter, least squares) may be used. For continuous position estimation, the algorithm to estimate position, velocity and time (PVT) is usually a Kalman filter $[29,55]$.

\subsection{WLAN}

As mentioned, GNSSs are studied and improved since thirty years [15], whereas localisation with help of WLAN signals is a relatively recent field [3,23]. In the last years several techniques have been investigated. Different localisation techniques lead to different advantages and drawbacks.

For lateration, the positions of the transmitters the WLAN access points (APs) - must be known, which they are not in usual, and precise time measurements are essential. Determining the APs positions may be difficult and moreover the APs must stay fix at known positions. These requirements are hard to fulfil in uncontrolled areas. Furthermore, one has to deal with non-line-of-site conditions and with severe multipath effects, especially indoors [20]. Various time delay measurement methods, for an overlaid positioning system based on WLAN, were investigated in $[2,21,35,74]$.

A second geometrical method is angulation. Instead of time delays, angles are measured and used to derive a position. Angulation suffers from similar drawbacks as lateration. These are mainly the unknown and possibly variable positions of the APs and non-line-of-sight conditions. Triangulation with IEEE 802.11 signals was studied in $[30,33,37]$, and might become important again with further deployment of the IEEE 802.11n standard and antenna arrays in mobile devices. [31] proposed a WPS relying on beamforming, that estimates the mobile device position from WLAN signals received from a transmitting beamformer, whose location and calibration pattern is known.

The stated problems of the geometrical methods are still not satisfactory solved. Some of these approaches need hardware and/or software modifications of the $\mathrm{Wi}-\mathrm{Fi}^{\mathrm{TM}}$ equipment to work, other diverge from the IEEE 802.11 standard [2]. Besides the timing issue, the unresolved problem of multipath propagation, diminishes the accuracy of these systems further. Therefore, most WPSs use received signal strength measure- 
ments (RSS) to estimate a position. Based on RSS, either i) lateration or ii) pattern recognition of RSS is employed.

In i) a distance between the WLAN transceiver and an AP is deduced from the RSS via modified free space propagation models. This was also already suggested in [3] and then further adapted to different scenarios by many other researchers. The position can be estimated via trilateration with at least three distances. In realistic urban and indoor environments the IEEE 802.11 radio channel is influenced by several independent causes and varies in time [24]. Resulting in a complex signal propagation, which makes signal strength measurements at a certain point in space hardly predictable [5, 44]. Although the modelling of that IEEE 802.11 channel characteristics makes progress, these models still lack of precision to be universally applied for positioning in urban canyons and indoors.

The second technique to employ RSSs in ii), is to use location fingerprinting. Location fingerprinting is performed in two phases: an off-line and an on-line phase. First, RSS measurements of all receivable APs are recorded at known reference positions of the target area. These signal strength measurements and their according reference positions are stored as fingerprints in a data base, often referred to as radio map. ${ }^{1}$ During the on-line phase RSS observed at a certain location are compared against the data base entries from the off-line phase. For this comparison various pattern recognition techniques with many variants are available, see $[26,38]$. The best matches of that comparison indicate the most likely locations.

The alluded problems with WLAN ranges, either obtained from time measurements or from signal strength measurements, lead to a lack of robustness against variations of the environment, which in comparison WLAN RSS fingerprinting does not show. A general overview of positioning in wireless networks can be found in $[56,62,69]$.

Above all, a WPS may compute its position estimate on the mobile device such as a GNSS receiver. So, by combining these to positioning techniques appropriately, as well privacy concerns are almost without cause. Disadvantageous and costly is the recording of the radio map. For large areas it is a very time consuming process. In addition it must be

\footnotetext{
${ }^{1}$ In WLAN fingerprinting the radio maps can be stored directly on the mobile device, but due to the size of target areas the radio maps are often downloaded via a data connection when the user comes in the vicinity of the building or the target area.
}

maintained and updated from time to time to adapt for changes in the WLAN infrastructure and environment.

\subsection{Hybridisation}

With hybridisation it is referred to a combination of different information, in other words a fusion of measurements from a variety of sensors. Measurements in this case is sensory data or data derived from sensory data. It is expected that the fused data is enhanced and more informative. Data of different systems can be combined at different levels: i) direct fusion of sensor data, if the data are commensurate, for example sensor raw data of the same sensors; ii) fusion of feature vectors, representations of sensor data; or iii) a fusion of high-level inferences or decisions based upon information from sensor data, compare [36]. In general, raw data fusion is more accurate than featurelevel or decision-level fusion, because some potential useful information is lost in the processing of sensor data or is only approximated by that processed data [22].

\section{Review of hybridisation systems}

When information from GNSS receiver is fused, to kinds of GNSS data is used: pseudo ranges or the GNSS receiver position itself. ${ }^{2}$ Whereas on WPS side, due to the variety of position estimation techniques, much more different information for data fusion is available. This diversity of information and the level at which the information of the both systems is integrated, leads to a large number of data fusion approaches of GNSS and WPS. In the following a categorisation of the existing hybridisation methods for GNSS and WPS is intended. Note that the data fusion algorithms working on raw data may additionally deduce features from sensor data and hence may be assigned to several categories. Like this, the sought quantity can be determined when for instance a data source is not available or lacks of integrity, which makes these methods more robust. The approaches are sorted from less complex integration techniques on decision-level information to data fusion algorithms, which integrate the information deeper via filtering techniques.

\footnotetext{
${ }^{2}$ An exception is a deeply coupled GNSS/INS integration, where tracking loops of the GNSS receiver are controlled by information about the system's dynamic obtained from inertial sensors.
} 


\subsection{WLAN assisted GNSS}

The time needed to compute the first position after the start up of a GNSS receiver is called time-tofirst-fix (TTFF), and systems reducing that time are so called assisted GNSS (A-GNSS). The use of a WLAN for this purpose is twofold and is proposed in [71] and [72]. One purpose is to provide a coarse initial position, the other is to establish a communication connection. The two main factors for a long TTFF, are the acquisition of satellites and the demodulation time for the ephemerides [29].

GNSSs are based on a CDMA scheme, which is used to estimate the signal transmission time and to identify the different satellites. Therefore, the transmitted code must be aligned with a local replica of that code. To accomplish this alignment, the delay of the transmitted code with respect to the local code and the delay due to the Doppler shift must be known. Thus, acquisition of a GNSS signal is to search the complete frequency and code delay space, which is normally quite costly. In an assisted GNSS an initial, coarse position is provided to narrow down that search space, and substantially reduce the acquisition time. Such an initial position can be provided by a positioning system relying upon WLAN infrastructure. The initial position can be used by the GNSS receiver to search directly for the satellites in view instead of searching all satellites. The reduced search space allows additionally a much narrower signal search bandwidth, which enhances the sensitivity of the GNSS receiver. Especially in high density urban environments this gain is beneficial.

The second factor increasing the TTFF is the demodulation time of the navigation messages. If a GNSS receiver performs a "cold start" it takes several minutes to obtain the first position estimate. In the case of GPS with a data rate of $50 \mathrm{bps}$, a GPS receiver requires up to 12.5 minutes to receive the ephemerides necessary to compute the first position fix. Via an established WLAN communication link, the already decoded GNSS ephemerides and satellite clock correction data can be downloaded much faster than via the GNSS satellite link and hence the TTFF in a "cold start" would be reduced considerably.

\subsection{Switching between GNSS and WPS positions}

The most common and most intuitive procedure to combine measurements from a GNSS receiver and measurements from a WLAN transceiver, is to use the position derived by each system independently. It can be distinguished between methods which only switch between the positioning systems, and methods which actually fuse positions to one position, see Section 3.3. Usually it is switched from one positioning system to another positioning system, because the former system is not able anymore to derive a position or a more accurate position is available from the second system. Also the last smart phone generations are able to provide a somehow derived position ${ }^{3}$ as an alternative to a GPS position and can switch between these positioning modes, see application programming interfaces (APIs) of modern cellular phone operating systems. Notice, modern mobile devices may also be able to integrate GNSS and WLAN positioning techniques deeper with help of the Skyhook Wireless hybrid positioning system called XPS. Skyhook Wireless cooperates with global players GNSS/Wi-Fi ${ }^{\mathrm{TM}}$ chip producers, whose chips can be found in the majority of mobile devices and likely enable this hybrid positioning system for many users [61]. Further details about the XPS hybridisation method are not disclosed. Different information about the localisation method can be found in the literature. That the Skyhook WPS relies on fingerprinting is stated by $[17,79]$, that it is based on multilateration can be found in $[43,66]$. The fact that Skyhook's web site notes a "massive reference data base of $\mathrm{Wi}-\mathrm{Fi}^{\mathrm{TM}}$ access points" suggests the use of lateration.

The most general procedure to switch between different positioning sources is obtaining a GNSS position if the user is outdoors (GNSS detectable), and obtaining a WPS position when no GNSS position is available, usually indoors. If none of these two systems is available and the device is equipped with a modem for cellular networks, a position is obtained from that. Unfortunately it is not clear to all details how a position in mobile devices is finally deduced. The GNSS receiver is in most cases a consumer grade A-GPS receiver. About the WPS is less disclosed and it is probably carried out differently in each mobile device operating system (OS).

Building up on one of the mentioned APIs and their location frameworks, various location aware applications have been proposed. A few of them deal in particular with a seamless indoor/outdoor positioning. In [49], for example, the Symbian Mobile Location Framework makes use of positions from an inter-

\footnotetext{
${ }^{3}$ This is usually a WPS and/or a cell-ID derived position, but the position may be obtained by something else. For example a pedestrian reckoning system relying upon accelerometer and compass.
} 
nal and an external GPS receiver, and from positions derived from a WPS and the cellular network.

The authors of [8] presented a multisensor positioning system suitable as well for collaborative localisation. Different position sources, amongst others AGPS and WPS, are all requested in parallel and for each an accuracy is deduced. All position estimates, which achieve a specific accuracy threshold are stored, and the position with the highest accuracy is chosen as the current device's position. Moreover, a devaluation function based on the position accuracy is defined to diminish the accuracy of the stored positions over time. A new position with an accuracy lying within the mentioned accuracy threshold, is then compared against the (devalued) accuracy of the current position. Only if the new position has higher accuracy, the current position of the mobile device is updated.

The position switching strategy described in [70] decides mainly on the availability of GNSS, WPS and cell-ID. The OS executes that task. In addition historical location information of the user and clustering of this data is incorporated into the algorithm. K-means clustering is used on the historical location data to condense the data sets and to compensate for outliers (different thresholds for WPS and GPS are applied). This data is then clustered to generate a personal mobility map, which helps to save energy and to refine the tracking accuracy of the mobile device.

Likewise the approach presented in $[47,48]$, where the location API of the OS is also used to switch between positions, and a mobility model is also employed to refine position accuracy. Besides, to decrease the energy consumption, additional sensors and algorithms are added. The radio frequency based positioning systems (GPS, $\mathrm{Wi}^{-\mathrm{Fi}^{\mathrm{TM}}}{ }^{\mathrm{T}}$, GSM pos. system) of the OS are considered as expensive with respect to their energy needs. A personalised mobility model of the user, derived from former user positions, and an inertial sensor based positioning algorithm are augmenting the system. For each position providing system a position accuracy measure is derived, or obtained from the OS respectively. On the basis of this data the trade-off between energy consumption and position accuracy is made. In both of the last presented approaches the localisation improvement is achieved by applying transition probabilities to the mobility models.

The majority of applications relying upon a location framework of an OS, aim towards LBS and use these frameworks unmodified, so that we not further consider these approaches.
A position mode switching by solely checking the availability of GPS was presented in [76]. GPS is the main position provider. The system switches to the WPS if the GPS fix is lost, and switches back if the fix is available again.

A bit more advanced approach of integrating WLAN and GPS into one system is presented in [50,51]. Between three different localisation methods is switched automatically, dependent on the availability of these methods. One is called "place detection" and is a WLAN fingerprinting reduced to a set of common places. WLAN RSSs are used to recognise the current location among common known places. If the "place detection" does not recognise the user's location the system switches to GPS. Only when GPS can neither provide a position estimate it is switched to an other WLAN based method. A WLAN localisation algorithm based on a previously created data base of WLAN access points and their positions. This algorithm estimates the position as weighted mean of the positions of receivable APs. The weights are determined in terms of the RSS, the AP coverage and the total number of detection of the AP, which has been recorded while creating the data base.

Based on GPS and WPS, [23] discusses four different position switching strategies to deal with indoor/outdoor transitions. The GPS and WPS run continuously and keep sensing for signals to reestablish the position estimation. The finding of the paper is that the best handover strategy would be to prefer GPS until the position fix is lost for five seconds; then the WPS position is used until a GPS position can be obtained.

A similar method was published in [19], which additionally uses information of the radio map. In the radio map, certain indoor transition zones are marked where entrances are located. GPS is used outdoors. Is a transition zone is detected, the system switches to the WPS. It stays in WPS mode until no WPS derived position is reported for a few seconds, then it switches to GPS to acquire satellites. If indoors, the systems also switches from WPS to GPS when a user position is within a transition zone for a given time. If a change of positioning mode is initiated, but signals of the other positioning system are not detected, the algorithm switches back to the previous mode.

\subsection{Weighting of GNSS and WPS positions}

As in the section before, the hybridisation methods presented here derive WPS and GNSS positions independently, but only one position is provided to the sys- 
tem. Features are combined instead that just a decision between two positions is made. The algorithms which will be described, weight positions derived from different sensors, to provide a common position, according to application specific criteria. In the data fusion process additional information is taken into account, to combine several positions to one, possibly more accurate and robust, position. To accomplish the fusion, some of the following approaches use either simple weighting strategies or adaptive filters. Data fusion methods based on adaptive filtering can usually mathematically described by the recursive Bayesian framework. This framework is a general, probabilistic description of a state estimator, providing an estimate of an internal state obtained from noisy measurements. It involves a process model, describing the physics of the system evolving in time, and an observation model, describing the measurement process. The Kalman filter (KF) was a long time the standard method to fuse different measurements, especially applied for target tracking. The KF assumes linear processes with Gaussian noise and hence is able to approximate the involved probability distributions with their first and second order moment. (The KF performs a linear combination (related to weighted mean), where the weights are reasoned from the measurement covariances.) The Kalman filter applies for linear, Gaussian models since under these conditions the distributions remain Gaussian. If these conditions are not met, modified versions of the Kalman filter apply. The extended Kalman filter (EKF) linearises about an estimate of the current first and second order moment, but is only valid for Gaussian noise. For highly nonlinear processes the unscented Kalman filter (UKF) is often the filter of choice. Instead of approximating the non-linearities, the UKF approximates the probability distributions of the Bayesian recursion with samples, which capture the first two moments of the distributions. This approximation is accurate up to the third moment for Gaussian distributions and accurate up to the second moment for non-Gaussian distributions. In a likewise fashion the cubature Kalman filter (CKF) approximates the probability distributions functions, but the sample points are chosen corresponding to a cubature rule. The CKF will not be further explained, since it found no application in the reviewed publications. A filter used widely in localisation and tracking applications is the particle filter (PF). The PF approximates the unknown probability distributions with a set of samples and associated weights, allowing the approximation of arbitrary probability distributions. Due to this compelling property, it is kind of all-purpose. This advantage comes on much higher computational costs compared with the KF variants.

Algorithms based on rather simple weighting strategies will be presented first:

In [77], a weighting scheme was investigated combining outcomes of a WLAN and GNSS location fingerprinting algorithm. In the on-line phase GNSS positions (longitude, latitude) and WLAN RSS are stored in data bases. To increase robustness of the fingerprinting methods, for each GPS fingerprint, the GPS coordinates are recorded at three different weather conditions; for each WLAN fingerprint, the WLAN RSS are recorded in the four cardinal directions, which are then averaged. The position estimate for both sources is obtained by standard fingerprinting algorithm based on the Euclidean distance. The weighting of the two positions is achieved by the linear combination $p=$ $(1-\beta) p_{\mathrm{WPS}}+\beta p_{\mathrm{GPS}}$ dependent on the weather conditions. The weight was found empirically: For sunny $\beta=1$, when cloudy $\beta=0.9$, and $\beta=0.7$ for rainy conditions. To estimate the proper user position the algorithm needs the input from the user about the weather condition. These developments have been extended with a Zigbee position fingerprinting system and a different weighting algorithm [78]. In this approach, three data bases for three different positioning systems must be recorded in the off-line phase, and three different positions are estimated in the online phase. These three position estimates (if available) are finally linearly combined via $p=\alpha p_{\text {WPS }}+$ $\beta p_{\mathrm{GPS}}+\gamma p_{\text {Zigbee }}$. The weights are derived according to $\alpha=\frac{1 / \sigma_{\mathrm{GPS}}}{1 / \sigma_{\mathrm{GPS}}+1 / \sigma_{\mathrm{WPS}}+1 / \sigma_{\text {Zigbee }}}$ as example for GPS. The standard deviations are deduced from sample statistics. $\beta$ and $\gamma$ are derived by exchanging the numerator for $1 / \sigma_{\mathrm{WPS}}$ and $1 / \sigma_{\mathrm{Zigbee}}$, respectively. They further suggest to update the variances during the on-line phase to improve their method.

The algorithm described in [60], retrieves positions and additional information from a conventional GPS receiver and a WPS. Fingerprinting, with an algorithmic addition accounting for WLAN signal multipath effects, is applied to obtain a WPS position. Important to mention is that 3 different radio maps, one for indoor, one for outdoor and one for both (overall), are generated. This way undesired position jumps between indoor and outdoor can be avoided, if the presence of the user in the indoor or outdoor area is correctly detected. A linear weighting scheme is presented in that work, relying mainly on the availability of the 
GPS. The GPS and the WPS positions are combined by $p=(1-\beta) p_{\mathrm{WPS}}+\beta p_{\mathrm{GPS}}$. The parameter $\beta$ is set according to the following cases: a) no satellite in view, $\beta=0$, and a WPS position is derived from the indoor radio map; $b$ ) the number of satellites is between one and four, $\beta=0$, and a WPS position from the overall radio map is derived; c) more than 4 satellites are available but one of the GNSS signals has very low signal to noise ratio, again $\beta=0$, and a WPS position from the outdoor radio map is computed; $d$ ) number of satellites is larger 4 and all GPS signal to noise ratio conditions are fulfilled, the WPS position is derived from the outdoor radio map, and is linearly combined with the GPS position. In this case the geometrical dilution of precision (GDOP) decides if $\beta$ equals $0.25,0.5$ or 1.0 .

Algorithms relying upon adaptive filtering will be presented in the following:

A multisensor data fusion method integrating GPS and WPS by means of a Kalman filtering was proposed in [32]. The focus of this work lies on the integration of the multiple sensors, and in particular on camera image processing to stabilise the heading estimation. Besides inertial sensors, a compass and a camera are used for a dead reckoning (DR ${ }^{4}$ system, and Bluetooth, WLAN and GPS serve with an absolute location estimate to the system. The positions from the GPS and the WPS contribute to the final position estimate via the KF equations. Their weights are influenced by the predicted state and the measurement noise covariances trough the Kalman gain.

[12] presents a position fusion method, based upon Kalman filtering and an interacting multiple model (IMM) estimator. The used positioning systems are GPS and awiloc ${ }^{\circledR 5}$. The IMM usually employs multiple Kalman filters with different process models, each accounting for a different motion the mobile device may perform. It combines the output of the filter adaptively by computing a weighted sum. The filters run in parallel and conditional probabilities are computed, which mix (weight) the outcomes of the two filters in an optimal manner. The weights are mode probabilities deduced from the fact how well fits the data propagated through the process models to the last measurements [4]. In [12] the IMM is used differently. Instead of a weighting according to the performed motion, the process model is the same for both filters and the IMM

\footnotetext{
${ }^{4}$ Dead reckoning is about estimating the current location based on motions made since the last known position. The motion is in general approximated by velocity and course estimates.

${ }^{5}$ A commercial WPS employing RSS fingerprinting [1].
}

algorithm is used to weight according to the quality of information (QoI) of the GNSS and WPS position estimates. In the case of GNSS this quality measure are reasoned from the GDOP and awiloc ${ }^{\circledR}$ delivers its proprietary error measure.

An approach to fuse GNSS and WPS incorporated into a DR system for vehicle localisation was proposed in [73]. The system design consists of a federated KF. The DR system, consisting of a gyroscope and an odometer, estimates within an UKF the state of the vehicle. To bound the error grow of the DR system, absolute positioning sources are loosely coupled with the DR system. In parallel to the UKF a KF is set up to fuse GPS and WPS positions. The integration of the UKF and KF outputs happens in a federated form. A master KF combines the states of both subfilters and feeds the final estimates back to the two subfilters. The WPS takes the role of the GPS in GPS denied environments. It helps to limit the error accumulation of the DR and provides an absolute initial position.

As well $[41,42,45,46,68]$ use a WPS to back up the GNSS component. In these publications, a high sensitivity GNSS receiver is investigated, to overcome the localisation problems in high rise urban areas and eventually indoors. Remind the CDMA scheme for GNSS signals, with signal power levels around the noise floor. Obstacles such as buildings and so forth impair this situation further, which then makes a signal acquisition and tracking with a conventional GNSS receiver unfeasible. To still acquire and track GNSS signals, the suggestions of the previously cited references, are to increase the integration time and to decrease the tracking bandwidth. On that account, nonetheless further sensors are necessary for a HS-GNSS receiver design. Inertial sensors are employed, to deliver information about the dynamics of the mobile device. In addition, inertial sensors provide opposed benefits and drawbacks compared to GNSS. When combining the two systems, the GNSS bounds the errors of the inertial navigation system (INS) and calibrates the inertial sensors; whereas the INS bridges GNSS outages and smooths the GNSS solution. The fusion architecture in $[41,42,45,46,68]$ is a deeply integrated INS/GNSS with aid of further sensors. Since the application aims at mobile devices, only micro-electromechanical system (MEMS) inertial sensors are qualified. INS/GNSS yields a power gain, because not the whole absolute dynamic of the antenna (mobile device) must be tracked, but only the INS errors. Thus, a lower tracking bandwidth is required, which increases the noise resistance; due to enabling a longer integration time. The 
inertial sensors data helps during highly dynamic motion to keep the tracking loops in lock. Furthermore, a deep coupling enables the INS/GNSS for short periods to work with less than four satellites. The INS is vitally to a HS-GNSS, but without corrections from GNSS it can not support the system for a long amount of time (dependent on error growth). WPS comes into play when nevertheless the GNSS looses its position fix. A WPS position is then used to provide a position to limit the errors of the INS. Among other sensors, WPS is fused into the system as a KF position measurement.

In the work of [39] as well a HS-GNSS receiver is developed, following a very similar strategy: a HSGNSS receiver based on a INS/GNSS tight integration scheme with a WPS among other sensors. Again, the WPS supplies an independent, absolute position to the EKF, and in particular to the INS. Only with an additional position input the INS error can be kept in reasonable limits, during GNSS outages. For further details on HS-GNSS and INS/GNSS and the different integration algorithms, it is referred to $[29,64]$.

Also a method using a particle filter to combine the two positioning systems has been published. The following two approaches [58,59] describe primarily a WLAN RSS fingerprinting particle filter. Their contribution to data fusion of GNSS and WPS is to insert sporadic occurring GPS fixes in their positioning solution. In this approach the GPS position fixes are assumed to be of better accuracy than the WPS position estimates. When a GPS fix is available the particle cloud becomes re-centered at the GPS location and the weights become normalised.

The already briefly mentioned hybrid indoor positioning approach proposed in [31], makes use of a particle filter too. Even though GNSS was not used in their experiments, they already had in mind to accommodate the system for outdoors and therefore integrate a GNSS receiver. Their method integrates angle measurements from WLAN signals and pedestrian DR, by employing a particle filter and map filtering.

A geometric approach, improving the WPS accuracy by solely two GNSS satellite signals, is proposed in $[18,34]$. The key idea is to use the pseudo range difference of two satellites to eliminate the GNSS receiver clock error, and so to narrow down the possible area of the receiver location. The difference of two ranges give one hyperboloid surface, which intersects in a line with the earth's surface. One point of this line is the possible receiver location. To find the receiver location on that line a WPS position, or an other external position provider can be exploited. By simply deriving the shortest distance from the WPS position to the line of candidate positions, the receiver location can be obtained. Notice, pseudo ranges are observed, which contain several errors. Hence the intersection line is actual a intersection stripe, making the result less reliable. Relying upon lateration, the accuracy of the intersection line depends heavily on the satellite geometry. The larger the angle between the two satellites, the more accurate the intersection line of the TDOA hyperboloid and the spheroid approximating the earth.

\subsection{Integration of GNSS pseudo ranges with WPS features}

In contrast to the former section, now data fusion architectures are considered, which may not provide positions independently to the application, but deduce a common position from features derived from sensory data. To provide robust systems, able to cope with signal outages and so on, a single subsystem ought to be able to compute a position on its own. The majority of the approaches, presented in the following, make use of lateration. GNSS pseudo ranges and ranges from WLAN signals are combined in a way that WLAN ranges complement the GNSS PVT equations. It is worth noting, that WPSs are in most cases software solutions, more or less easy to modify to obtain the desired sensory data. Whereas the GNSS chip in the systems, described in the following, must be able to deliver pseudo ranges.

Due to imprecise timing of WLAN chips and missing synchronisation of the network, accurate WLAN ranges are not easily obtained directly by measuring time delays [21,35]. An other difficulty when measuring transmission times, is that the positions of the WLAN APs must be known, which are usually not. As well problematic in unknown, uncontrolled environments is that APs can easily be moved, vanish, reappear, etc. Multipath propagation is another drawback, which has to be overcome for an accurate positioning based upon time measurements. Many of these problems have not been solved yet or could only be solved by modifying hardware or firmware. No development is known to the authors which has solved all, for its approach specific, problems. WPSs RSS fingerprinting solutions on the other hand are much more widespread.

The authors of [80] addressed hybrid localisation in rural and suburban environments. In their approach pseudo ranges from GNSS satellites are complemented with ranges deduced from WLAN RSS. The position 
is estimated by lateration. They employ three subsystems: first a GNSS receiver estimating a stand-alone position, when more than 4 satellites are detectable; second a WPS, estimating a stand-alone positions from WLAN RSS deduced ranges, if more than four APs are detectable. A third module, fuses range measurements of both subsystems. In that third module pseudo ranges and WLAN ranges solve the localisation problem, when both systems together provide at least 4 ranges. The system may have three positions from the three modules available. One from the GNSS receiver, one from the WPS and one from the fusion module. The final position estimate is a combination of the possibly three positions based on the combined GDOP derived from all (pseudo) ranges.

In [14] as well the combination of pseudo ranges and WLAN ranges is investigated, but focused on the case of less than four satellites in view. The work concentrates on the issue, which exists more likely when ranges of very different magnitudes are fused: ambiguous position solution. The position solution of lateration may be ambiguous, due to coplanar transmitters. In GNSS this is usually not a problem, because the ranges to the satellites are huge and one of the solutions does not lie on the earth's surface. Thus, the solution far away from the earth can be excluded. The problem of ambiguous solutions occurs more likely when much smaller WLAN ranges and pseudo ranges are in a common set of lateration equations. Two valid position solutions are almost always derived in the common case of combining three pseudo ranges and one WLAN range. In [14] a method is developed to solve that ambiguity by exploiting information about the GNSS and WPS positions gathered over time. The algorithm relies on the key idea that in a static receiver scenario the false position must move due to the movement of the satellites and the true position solution must not; but since the measurements contain errors, both move. So, the two possible solutions are derived with the Bancroft algorithm. Furthermore, the evolution over time of these two initial positions is stored for four minutes and compared against the initial positions. The evolution of true initial position is supposed to have a smaller slope, hence it deviates less over time than the wrong position.

A combination of almost all so far presented ideas to fuse GNSS and WPS can be found in [75]. The algorithm of [75] first switches between four modes dependent on the availability of GNSS and WPS, and the number of receivable GNSS signals. Then it uses, according to the chosen mode, an adaptive filter to fuse features of the two systems. In accordance with the four conditions, the following mode and positioning algorithm are chosen: a) WPS but no GPS, WPS standalone and least square method; b) WPS and GNSS but with less than four satellites, KF to fuse GPS and WPS ranges; c) WPS and GNSS with more than four satellites, federal KF to fuse positions derived by each subsystem; and d) GPS but no WPS, least square or KF (as usual for GNSS). Details about the design of the filters or how the different filters are used in particular is not revealed.

A last approach fusing GNSS pseudo ranges and WPS features is a probabilistic algorithm proposed in [52]. A particle filter is applied to merge likelihood functions, obtained from GNSS pseudo ranges and WLAN RSS pattern recognition. The pseudo range likelihood function is modelled as a Gaussian distribution and is derived from available pseudo ranges. The derivation of the likelihood of the RSS measurements is not that straight forward. Recall the RSS pattern matching of the on-line phase, where a current RSS observation is compared with the RSS data base entries (recorded during the off-line at known reference points). The outcomes of the RSS comparisons usually weight the according positions to obtain the final estimate. To fuse the likelihoods, in [52] the RSS comparisons are not done with the fingerprint positions in the data base but with the particles. An approximation is carried out to map RSS data base entries from fingerprint reference points to particles. By normalising the weights obtained from the data base comparison, such that the weights sum up to one, a pseudo-likelihood over the target area is established. The GNSS likelihood is represented by particles and corresponding weights and the WPS pseudo-likelihood is represented by the same particles and weights derived from the data base comparison. Multiplication of the likelihood functions accomplishes the data fusion.

\section{Performance comparison}

First of all we must state that an absolute, objective evaluation of the presented methods, based on the reported experimental results, is not possible. Too different are the applications and objectives of the studies, and too different are the development stages and the scenarios of many of the approaches. Some approaches are already quite mature, are tested and improved several times, whereas others are solely analysed on simulated data. If tests in real environments were con- 
ducted, the scenarios differ significantly. Not all considered their system as ubiquitous and performed indoor and outdoor tests. Several approaches are constrained to vehicle motions, others are more specific for pedestrians. Hence, it can only be tried to compare the approaches qualitatively. Accuracy, precision, robustness, complexity and costs will be evaluated. Resting on the depth of the data fusion, each category is surveyed towards its potential performance. To have a common understanding of these criteria, we follow the remarks of [38] and [63] on performance of positioning systems.

Accuracy describes how good a location system performs, the metric representing the accuracy is mostly the average euclidean distance. The better the accuracy, the smaller is the deviation from the ground truth. A bias or a systematic influence are considered within the location error.

The precision in contrast, represents the consistence of a positioning system. It contains information about the variation in its performance over several trials. A measure to capture the precision is the cumulative distribution function (CDF) or percentiles.

With robustness it is referred to how likely a positioning system keeps functioning under harsh radio frequency signal conditions, for example when signals are totally or partially blocked, or have low power. It depends strongly on the environment and the variation and modifications of the environment. Robustness is also linked to coverage, continuity and availability. In fact in the context of GNSS and WPS fusion, these criteria are determined in first place by the deployment of WPS enabled areas. Meaning the arrangements of target areas to localise with WLAN location fingerprinting or WLAN ranging; which is not covered herein.

The concept of complexity can be applied at many stages, such as hardware, firmware and computational complexity. Here, primarily computational/algorithmic complexity is considered, even though for some WLAN ranging methods hardware, or firmware modifications are necessary. Last but not least costs are examined.

Costs are understood very broad. Certainly, costs are also closely connected with complexity. For example, monetary costs increase with modifications of hardware, and battery consumption increases with computational complexity. This paper focuses mainly on battery drainage, because the majority of algorithms aim for mobile devices and energy consumption is always a critical issue. Costs of infrastructure are not further considered in this comparison, because GNSS and a WPS enabled environment is required for all approaches.

\subsection{WPS aided GNSS}

Recall the references in Section 3.1, dealing with WPS aided GNSS. Envisaging solely the integration of the WPS position, the overall accuracy and precision is not improved.

One could state, that because of an earlier GNSS fix the robustness is increased somehow.

The whole method to decrease TTFF is rather complex. But again, considering only the fusion of the WPS position to narrow down the acquisition search space and not the possibilities coming with the data link, the complexity is only increased slightly.

The power consumption of the WPS comes in addition to that of the GNSS receiver, which increases the costs. By contemplating to use all kind of aiding data, accuracy, precision and robustness improve and the system complexity increases by the WLAN data connection. Then the battery drainage may be reduced, in particular in the cold start phase, because the acquisition time is reduced and a WLAN connection needs usually less power than a GNSS receiver.

\subsection{Switching positions}

In the case of the position switching methods, presented in Section 3.2, the overall system accuracy and precision can not be better than that of one of the standalone subsystems.

It can be surely stated, that the combined system is more robust, gaining from different enabled localisation environments for WPS fingerprinting and GNSS. A much wider coverage area, due to complementing systems, is the main aim of the data fusion of WPS and GNSS.

The complexity in terms of the algorithm is almost not worthy of mention. In most systems presented, switching the positioning mode is just to switch on/off a subsystem, reasoned from its availability or a parameter comparison with a, for instance, battery level threshold or accuracy thresholds.

Concerning the consumption of energy, two cases must be distinguished. On the one hand, when only the selected system is running and on the other hand, when WPS and GNSS (and possibly more) systems run in parallel. In the latter case the battery drainage may be more than doubled in comparison to a stand-alone positioning system. In the other case it would be the same compared to the stand-alone system. 


\subsection{Fusing positions}

To evaluate the performance of the approaches of Section 3.3 , one should bear in mind that these approaches weight positions to a common position by means of linear combination or adaptive filters. All these approaches have in common that the position is only combined properly, when the QoI about the single position estimates are significant. With a quality of information, or sometimes also called quality of service $(\mathrm{QoS}){ }^{6}{ }^{6}$ in the context of this paper an indicator is meant, that reflects the errors of the position estimates.

In general it is quite difficult to model and detect all major error sources on position level. For example, the output of a low cost GNSS receiver does not provide overmuch information about the position quality. Parameters often used to assess the position quality are: the number of satellites, the carrier to noise ratio and DOPs (mainly GDOP and HDOP). They indicate a potential downgrade of the final position estimate, but are not reliable, and do not incorporate errors as for instance multipath errors. Moreover, many of these parameters included in the standard NMEA messages are correlated with each other, like the number of satellites and the DOP. Only a few of the plenty of them may provide useful information. Above all, the algorithms of commercial GNSS receivers are not disclosed and it is challenging to estimate an appropriate error measure after the signal processing chain of a GNSS receiver. Admittedly, enhanced GNSS receiver provide more meaningful error measures in their proprietary messages, which may the future low cost receiver too. As well for WPS holds, the evaluation of the quality of a position estimate is easier when the algorithms can be accessed, and additional information than just the position is provided.

When speaking about the accuracy of the systems presented in Section 3.3, it must be mentioned that the fused position can not be more accurate than the position computed from the sensor with the highest accuracy.

But in contrast to the former Section 4.2, the fusion of GNSS and WPS positions may improve the precision. The cause is a possible compensation of different random errors. Essential again, are the measures assessing the quality of the position estimates. Because of the error models often incorporated in the adaptive

\footnotetext{
${ }^{6}$ The term quality of information is preferred here, because QoS is often related the positioning service of the overall localisation system.
}

filters, their outcome is likely to be more precise than that of the simple weighting approaches.

The robustness of these position fusing approaches is of similar level as for the switching systems. GNSS covers outdoor environments and with help of a WPS the remaining areas may be covered. Such systems are much less prone to outages, and thus more robust than GNSS or WPS solely.

Compared to approaches with a switching strategy the complexity is increased. This increase is much higher for the adaptive filtering techniques than for the linear weighting schemes.

To fuse the positions of both subsystems, both systems must be active at the same time. This consumes more energy and increases the costs.

\subsection{Lower level feature fusion}

The potential performance of data fusion schemes based upon pseudo ranges and WPS features (Section 3.4) is still higher. But so are the complexity and the costs.

When fusing information on data level, a less processed level, more meaningful information can be retrieved from the signals. That assists evaluating the quality of data. Accessing the pseudo ranges allows integrity monitoring (e.g. RAIM) or a multipath mitigating strategy. When using a Kalman filter for the PVT computation, the innovation of the filter may provide some useful information with respect to errors and outliers. Having access to the fingerprint data base of a WPS, knowledge about the actual number of APs, etc., used for the position estimation, may help to get a useful QoI. Information about the number of recently appeared and unknown APs and the number of disappeared APs may also help to evaluate the reliability of the position estimate. Integrating this kind of information into the data fusion of GNSS and WPS, faulty measurements can be excluded from the position estimation. Thus, the accuracy can be significantly increased. Where on the other hand, the position fusion systems risk greater errors caused by false detection.

The uncertainty of measurements can be modelled more appropriate, may be according to certain environments, frequent scenarios or different motions. The probabilistic models for pseudo ranges usually follow a Gaussian distribution [29], which is justified with overbounding [53]. RSS measurements are often modelled as Gaussian distributed too, but this rarely fits well the measurements $[27,28]$. An access to the al- 
Table 1

Overview about the performance gain of different GNSS and WPS data fusion levels compared with stand-alone systems

\begin{tabular}{lccc}
\hline & accuracy & precision & robustness \\
\hline WPS aided GNSS & $0^{7}$ & 0 & complexity \\
GNSS-WPS Position Switching & 0 & 0 & + \\
GNSS-WPS Position Fusion & 0 & + & + \\
GNSS-WPS Feature Fusion & + & ++ & + \\
\hline
\end{tabular}

gorithms allows to refine the noise models, which can improve the precision of position estimates.

By feeding features of GNSS and WPS into a common position estimation process, less signals from one of the subsystems are required to compute a position. This makes the overall system much more robust and increases its coverage. Otherwise only one positioning subsystem or none at all would be usable.

Indeed, those approaches, like particle filtering, are much more complex and costly. Both subsystems are supposed to operate in parallel. Power consumption is larger than that of all former approaches and the up to day mobile devices may reach their limits, when contemplating processing power.

Table 1 summarises the statements of this section, comparing hybrid positioning approaches based on the four data fusion levels with GNSS and WPS standalone positioning systems.

\section{Conclusion}

This paper surveys the current data fusion approaches to combine GNSS with WPS for seamless positioning. Different methods and approaches, in progress to realise a truly seamless and ubiquitous positioning system, are presented. The great number of different approaches made it necessary to present the approaches in an ordered manner, according to the level information fusion taking place. Beginning with approaches using information of one positioning system to assist the other system in a specific task, over algorithms fusing data shallow on a decisionlevel, onwards to approaches actually combining location information, ending with dedicated algorithms to fuse information on data level. Rested upon this categorisation, the potential performance of these approaches is evaluated. The evaluation is done with respect to accuracy, precision, robustness, complexity and costs.

\footnotetext{
${ }^{7}$ Compared to the stand-alone systems, 'o' stands for no improvement and several ' + ' indicate a slight, up to a big improvement.
}

Concluding can be said that the performance in terms of accuracy, precision and robustness improves with the level of data fusion. As stated in Section 4.1, the idea addressed by the publications presented in Section 3.1, provides only a small gain with regard to robustness. A performance refinement is achieved by data fusion on a decision-level, compare Section 4.2. Systems where high level features are fused, as those presented in Section 3.3, can further improve the positioning performance (comp. Section 4.3). The integration of sensor raw data exploits the possibilities of a combined GNSS/WPS system to a greater extent and enhances the positioning performance even more (see Section 4.4). These improvements comes at the expense of higher complexity and resources as energy consumption.

\section{Future research and outlook}

The next crucial step towards an ubiquitous, more accurate positioning is a world wide unified WPS. Since, there exists nothing like a standard for WLAN positioning systems and GNSSs are explicitly designed to provide world wide location information, the most open issues are found on the aspect of WPS. Considering the advantages of WLAN location fingerprinting over WLAN ranging (higher accuracy, easy to implement, complement error to GNSS), and that it is widely spread and adopted by the majority of WPSs (particularly for indoor use), the future research based on location fingerprinting is illuminated in the following.

WLAN positioning systems which have been presented so far, were either quite accurate, aligned for localisation in a single or a few buildings, or aimed for large-scale localisation with an accuracy about $10 \mathrm{~m}$ at the best. This trade-off is caused by the off-line phase calibration overhead. Due to the progress in all adjacent fields (data storage, processing power, transmission bandwidth and so on) it is believed that WLAN location fingerprinting system can be large-scale and accurate. For a unified, ubiqui- 
tous WPS, one would need to agree on some principle points:

a) terminal-based or network-based,

b) a minimum set of data base entries,

c) a strategy to keep the radio map up to date and

d) a strategy to manage the vast data base.

This is only seen as a minimum accordance for such a world wide WPS. A lot of details are missing, of which some are discussed a little more in the following.

The system should not be owned and managed by a few participants [7], and therefore should be terminalbased. This decision additionally avoids a central control instance and privacy issues [57]. But on the other hand, the appropriate data base entries must be available on the mobile device or downloaded prior to estimate a position.

One must agree on a minimum set of entry types for the data base [26], on how to represent the location readings, units and the coordinate systems.

A scheme to manage such a large data base must be developed. For example a division in different hierarchies from country over state, city, district, building, floor to room could help to overcome potential stability issues. In addition, a strategy how to create, extend and update the data base must be developed, see for example [43]. It must be elaborated in a way not losing, better improving the accuracy over time. With a free access and an open interface, users and organisations might be encouraged to contribute and so to overcome the extensive calibration effort. The acceptance and the world wide availability, including (public) indoor areas and other not vehicle accessible areas, would grow faster. But as well technical tools might assist to improve the radio map creation. Studies using Gaussian process regression models showed that with just a few reference locations, used as training points, an accurate position estimation is possible, see $[6,11,16]$.

An agreement on how to compensate the different $\mathrm{RSSI}^{8}$ readings (e.g. [40,65,67]) from different IEEE 802.11 transceivers is necessary to achieve a consistent performance. Newer trends like the use of the $5 \mathrm{GHz}$ industrial, scientific and medical (ISM) band and multiple-input and multiple-output (MIMO) systems need be considered.

\footnotetext{
${ }^{8}$ Received Signal Strength Indicator shall emphasise at this point that these measurements are not deduced from a standardised relation between the RSSI and the automatic gain control. Such a relation does not exists, which makes calibration effort necessary.
}

Leaving future research fields of stand-alone WPS, robustness of hybrid systems is further increased by data fusion on at least pseudo range level. Because the position algorithm could operate with less then 4 satellites in view, beneficial especially in urban areas, where signal blocking due to high-rise buildings occurs often.

Consequently, GNSS receiver with pseudo range output must find their way into the future mobile devices. What is more likely are chips for localisation purpose with integrated GNSS receiver, WLAN transceiver and a processing unit, as already presented in [10]. Additional performance improvements are expected by further deployment of multi-constellation and multi-frequency receivers.

Many open fields of activity can be found in the information processing/data fusion area. In addition to a relatively high availability, which already the presented developments achieve, a tighter data fusion improves the accuracy and precision of the hybrid positioning systems. It facilitates further mutual advantages due to the complementary errors of GNSS and WLAN location fingerprinting. To decrease the common error further, advanced multipath mitigation schemes for GNSS and GNSS integrity monitoring, based on a WPS, should be incorporated into such a data fusion algorithm. Conversely, data from GNSS could improve the over all accuracy by testing the integrity of the WLAN measurements. Approaches to extract more information from pseudo ranges, WLAN ranges and WLAN RSS, useful to assess the quality of measurements, should be investigated more deeply. The more significant information is integrated into the modelling of uncertainty, the more the performance of the data fusion algorithms will improve. All this additional information give reason to employ more flexible Bayesian filters, for example histogram and particle filter. When dealing with heavy multipath propagation and cluttered measurements, the probability hypothesis density (PHD) filter might be applied.

What is more, such an accurate and reliable hybrid positioning technique, should be extended and employed to constantly update the WLAN location fingerprinting data base.

\section{Acknowledgements}

The authors would like to acknowledge the Mexican Consejo Nacional de Ciencia y Tecnología for the financial support for this work. 


\section{References}

[1] awiloc, awiloc $®, 2013$, URL http://www.awiloc.com, (visited on 19/02/2013).

[2] K. Bagdonas and K. Borre, Ubiquitous wifi/gnss positioning system - Toa based distance estimation, in: Proc. of the 21 st International Technical Meeting of the Satellite Division of The Institute of Navigation (ION GNSS 2008), 2008, pp. 17731779.

[3] P. Bahl and V.N. Padmanabhan, Radar: An in-building rf-based user location and tracking system, in: Proc. of Nineteenth Annual Joint Conference of the IEEE Computer and Communications Societies, INFOCOM 2000, Vol. 2, 2000, pp. 775-784.

[4] Y. Bar-Shalom, X.-R. Li and T. Kirubarajan, Estimation with Applications to Tracking and Navigation, John Wiley \& Sons, Inc., 2001.

[5] R. Battiti, M. Brunato and A. Villani, Statistical learning theory for location fingerprinting in wireless lans, Technical report, Informatica e Telecomunicazioni, Università degli Studi di Trento, 2002, URL http://rtm.science.unitn.it/ battiti/archive/ 86.pdf.

[6] A. Bekkali, T. Masuo, T. Tominaga, N. Nakamoto and H. Ban, Gaussian processes for learning-based indoor localization, in: IEEE International Conference on Signal Processing, Communications and Computing (ICSPCC), 2011.

[7] P. Bellavista, A. Küpper and S. Helal, Location-based services: Back to the future, IEEE Pervas Comput 7(2) (2008), 85-89.

[8] B. Bittins and J. Sieck, Multisensor and collaborative localization for diverse environments, in: Fifth UKSim European Symposium on Computer Modeling and Simulation (EMS), 2011, pp. 406-411.

[9] N. Bonnor, A brief history of global navigation satellite systems, J Navigation 65 (2012), 1-14, URL http://dx.doi.org/10. $1017 /$ S0373463311000506.

[10] csr, SiRFstarV ${ }^{\mathrm{TM}}$ architecture and SiRFusion ${ }^{\mathrm{TM}}$ platform, 2013, URL http://www.csr.com/products/138/sirfstarvarchitecture-and-sirfusion-platform, (visited on 17/05/2013).

[11] F. Duvallet and A.D. Tews, Wifi position estimation in industrial environments using gaussian processes, in: IEEE/RSJ International Conference on Intelligent Robots and Systems, IROS, 2008, pp. 2216-2221.

[12] D. Eck, K. Schilling, A. Abdul-Majeed, J. Thielecke, P. Richter, J. Gutiérrez Boronat, I. Schens, B. Thomas, B. Williger and F.R. Lang, Mobility assistance for older people, Appl Bionics Biomech 9(1) (2012), 69-83.

[13] N. El-Sheimy and C. Goodall, Everywhere navigation integrated solutions on consumer mobile devices, Inside GNSS 6(5) (2011), 74-82, URL http://www.insidegnss.com/node/ 2749.

[14] D. Fernandez, F. Barcelo-Arroyo, I. Martin-Escalona, M. Ciurana, M. Jofre and E. Gutierrez, Fusion of wlan and gnss observables for positioning in urban areas: The position ambiguity, in: IEEE Symposium on Computers and Communications (ISCC), 2011, pp. 748-751.

[15] C. Fernández-Prades, L.L. Presti and E. Falletti, Satellite radiolocalization from gps to gnss and beyond: Novel technologies and applications for civil mass market, P IEEE 99(11) (2011), 1882-1904.

[16] B. Ferris, D. Hähnel and D. Fox, Gaussian processes for signal strength-based location estimation, in: Proc. of Robotics Science and Systems, 2006.
[17] T. Gallagher, B. Li, A. Kealy and A.G. Dempster, Trials of commercial wi-fi positioning systems for indoor and urban canyons, in: IGNSS 2009 Symposium on GPS/GNSS, Citeseer, 2009.

[18] T. Gallagher, Y.K. Tan, B. Li and A.G. Dempster, Wi-fi+ gps for urban canyon positioning, in: Symp. on GPS/GNSS (IGNSS2009), 2009.

[19] T. Gallagher, B. Li, A.G. Dempster and C. Rizos, Power efficient indoor/outdoor positioning handover, in: Proc. of the International Conference on Indoor Positioning and Indoor Navigation (IPIN'11), 2011.

[20] S. Galler, J. Schroeder, G. Rahmatollahi, K. Kyamakya and K. Jobmann, Analysis and practical comparison of wireless lan and ultra-wideband technologies for advanced localization, in: Proc. of IEEE/ION PLANS 2006, Vol. 1, 2006, pp. 198-203.

[21] A. Günther and C. Hoene, Measuring round trip times to determine the distance between wlan nodes, in: Proc. 4th International IFIP-TC6 Networking Conference, 2005, pp. 768-779.

[22] D.L. Hall and J. Llinas, An introduction to multisensor data fusion, P IEEE 85(1) (1997), 6-23.

[23] R. Hansen, R. Wind, C.S. Jensen and B. Thomsen, Seamless indoor/outdoor positioning handover for location-based services in streamspin, in: Tenth International Conference on Mobile Data Management: Systems, Services and Middleware, MDM'09, 2009, pp. 267-272.

[24] A. Hatami and K. Pahlavan, Comparative statistical analysis of indoor positioning using empirical data and indoor radio channel models, in: 3rd IEEE Consumer Communications and Networking Conference, CCNC, Vol. 2, 2006, pp. 1018-1022.

[25] J. Hightower and G. Borriello, Location systems for ubiquitous computing, IEEE Computer 34(8) (2001), 57-66.

[26] V. Honkavirta, T. Perälä, S. Ali-Löytty and R. Piché, A comparative survey of WLAN location fingerprinting methods, in: Proc. of the 6th Workshop on Positioning, Navigation and Communication 2009 (WPNC'09), 2009, pp. 243-251, URL http://math.tut.fi/posgroup/honkavirta_et_al_wpnc09a.pdf.

[27] K. Kaemarungsi, Distribution of wlan received signal strength indication for indoor location determination, in: 1st International Symposium on Wireless Pervasive Computing, 2006.

[28] K. Kaemarungsi and P. Krishnamurthy, Properties of indoor received signal strength for wlan location fingerprinting, in: The First Annual International Conference on Mobile and Ubiquitous Systems: Networking and Services, 2004, MOBIQUITOUS 2004, 2004, pp. 14-23.

[29] E.D. Kaplan and C.J. Hegarty, eds, Understanding GPS: Principles and Applications, 2 edn, Artech House Publishers, 2006.

[30] K. Kawauchi, T. Miyaki and J. Rekimoto, Directional beaconing: A robust wifi positioning method using angle-of-emission information, in: Location and Context Awareness, T. Choudhury, A. Quigley, T. Strang and K. Suginuma, eds, Lecture Notes in Computer Science, Vol. 5561, Springer, Berlin, Heidelberg, 2009, pp. 103-119, URL http://dx.doi.org/10.1007/ 978-3-642-01721-6_7.

[31] P. Kemppi, T. Rautiainen, V. Ranki, F. Belloni and J. Pajunen, Hybrid positioning system combining angle-based localization, pedestrian dead reckoning and map filtering, in: International Conference on Indoor Positioning and Indoor Navigation (IPIN), 2010.

[32] H. Kuusniemi, L. Chen, L. Ruotsalainen, L. Pei, Y. Chen and R. Chen, Multi-sensor multi-network seamless positioning 
with visual aiding, in: International Conference on Localization and GNSS (ICL-GNSS), 2011, pp. 146-151.

[33] V. Lang and C. Gu, A locating method for wlan based location service, in: IEEE International Conference on e-Business Engineering, ICEBE, 2005, pp. 427-431.

[34] B. Li, Y.K. Tan and A.G. Dempster, Using two global positioning system satellites to improve wireless fidelity positioning accuracy in urban canyons, IET Commun 5(2) (2011), 163171.

[35] X. Li, K. Pahlavan, M. Latva-ahoand M. Ylianttila, Comparison of indoor geolocation methods in dsss and ofdm wireless lan systems, in: 52nd Vehicular Technology Conference, IEEE VTS-Fall VTC, Vol. 6, 2000, pp. 3015-3020.

[36] M.E. Liggins, D.L. Hall and and J. Llinas, eds, Handbook of Multisensor Data Fusion: Theory and Practice, 2nd edn, CRC Press, 2008

[37] C.-H. Lim, B.P. Ng and D. Da, Robust methods for aoa geolocation in a real-time indoor wifi system, Journal of Location Based Services 2(2) (2008), 112-121, URL http://www. tandfonline.com/doi/abs/10.1080/17489720802415189.

[38] H. Liu, H. Darabi, P. Banerjee and J. Liu, Survey of wireless indoor positioning techniques and systems, IEEE Trans Syst, Man, Cybern 37(6) (2007), 1067-1080.

[39] J.F.M. Lorga, P.F. Silva, J.S. Silva, T.F. Rocha da Silva, M.S. Nunes, F.D. Nunes and F.M.G. Sousa, Gnss hybridization for indoor positioning, in: Proc. of the 5th ESA Workshop on Satellite Navigation Technologies and European Workshop on GNSS Signals and Signal Processing (NAVITEC), 2010.

[40] G. Lui, T. Gallagher, B. Li, A.G. Dempster and C. Rizos, Differences in rssi readings made by different wi-fi chipsets: A limitation of wlan localization, in: International Conference on Localization and GNSS (ICL-GNSS), 2011, pp. 53-57.

[41] J.A. López-Salcedo, Y. Capelle, M. Toledo, G. Seco-Granados, J.L. Vicario, D. Kubrak, M. Monnerat, A. Mark and D. Jiménez-Baños, Dingpos: A hybrid indoor navigation platform for gps and Galileo, in: Proc. of the 21st International Technical Meeting of the Satellite Division of The Institute of Navigation (ION GNSS 2008), 2008, pp. 1780-1791.

[42] J.A. López-Salcedo, G.A. Vecchione, D. Kubrak, G. SecoGranados, J.L. Vicario, Y. Capelle, M. Monnerat, E. Aguado, P. Tiley, D. Palenzuela, M. Toledo, J.J. Rosales and D. JiménezBaños, Demonstration of ubiquitous positioning with wifi, ins and assisted hs-gnss hybridization, in: Proc. of the 4th ESA Workshop on Satellite Navigation User Equipment Technologies (NAVITEC), 2008, URL http://spcomnav.uab.es/docs/ conferences/DINGPOS_NAVITEC_2008.pdf.

[43] S. Meyer, T. Vaupel and S. Haimerl, Wi-fi coverage and propagation for localization purposes in permanently changing urban areas, in: Proc. of Wireless Applications and Computing and Telecommunications, Networks and Systems, IADIS Press, 2008, pp. 11-20, URL http://www.iadis.net/dl/final_uploads/ 200807L002.pdf.

[44] E. Mok, G. Retscher and L. Xia, Investigation of seamless indoor and outdoor positioning integrating wifi and gnss, in: XXIII FIG Congress, Germany, Ts 64, Munich, 2006.

[45] H. Niedermeier, B. Eissfeller, J. Winkel, T. Pany, B. Riedl, T. Wörz, R. Schweikert, S. Lagrasta, G. Lopez-Risueño and D. Jiménez-Baños, Dingpos: High sensitivity gnss platform for deep indoor scenarios, in: Proc. of the IEEE Int. Conf. on Indoor Positioning and Indoor Navigation (IPIN), 2010.
[46] T. Pany, J. Winkel, B. Riedl, H. Niedermeier, B. Eissfeller, T. Wörz, R. Schweikert, S. Lagrasta, N. Ricardo, G. LópezRisueño and D. Jiménez-Baños, Experimental results from an ultra-tightly coupled gps/galileo/wifi/zigbee/mems-imu indoor navigation test system featuring coherent integration times of several seconds, in: 5th ESA Workshop on Satellite Navigation Technologies and European Workshop on GNSS Signals and Signal Processing (NAVITEC), 2010.

[47] M. Papandrea, Multimodal ubiquitous localization: A gps/wifi/ gsm-based lightweight solution, in: IEEE International Symposium on a World of Wireless, Mobile and Multimedia Networks Workshops, WoWMoM, 2009.

[48] M. Papandrea and S. Giordano, Enhanced localization solution, in: IEEE International Conference on Pervasive Computing and Communications Workshops (PERCOM Workshops), 2012, pp. 241-246.

[49] L. Pei, R. Chen, Y. Chen, H. Leppakoski and A. Perttula, Indoor/outdoor seamless positioning technologies integrated on smart phone, in: First International Conference on Advances in Satellite and Space Communications, SPACOMM, 2009, pp. 141-145.

[50] L. Reyero and G. Delisle, A pervasive indoor-outdoor positioning system, Journal of Networks 3(8) (2008), 70-83, URL http://ojs.academypublisher.com/index.php/jnw/ article/view/03087083.

[51] L. Reyero and G.Y. Delisle, Positioning technologies for implementation of the always best located algorithm, in: IEEE Radio and Wireless Symposium, 2008, pp. 811-814.

[52] P. Richter, J. Seitz, L. Patiño-Studencka, J. Gutiérrez Boronat and J. Thielecke, Sensor data fusion for seamless navigation using wi-fi signal strengths and gnss pseudoranges, in: Proc. of European Navigation Conference (ENC), 2012.

[53] J. Rife and B. Pervan, Overbounding revisited: Discrete errordistribution modeling for safety-critical gps navigation, IEEE T Aero Elec Sys 48(2) (2012), 1537-1551.

[54] M. Sahmoudi, D.A. Grejner-Brzezinska, F. Gustafsson, G. Lachapelle, R. Landry and J.-Y. Tourneret, Introduction to the issue on advanced signal processing for gnss and robust navigation, IEEE J-Stsp 3(4) (2009), 537-540.

[55] N. Samama, Global Positioning: Technologies and Performance, Wiley-Interscience, 2008.

[56] A.H. Sayed, A. Tarighat and N. Khajehnouri, Network-based wireless location: Challenges faced in developing techniques for accurate wireless location information, IEEE Signal Proc Mag 22(4) (2005), 24-40.

[57] B.N. Schilit, A. LaMarca, G. Borriello, W.G. Griswold, D. McDonald, E. Lazowska, A. Balachandran, J. Hong and V. Iverson, Challenge: Ubiquitous location-aware computing and the "place lab" initiative, in: Proc. of the 1st ACM International Workshop on Wireless Mobile Applications and Services on WLAN Hotspots, WMASH'03, ACM, New York, USA, 2003, pp. 29-35, URL http://doi.acm.org/10.1145/941326.941331.

[58] Z. Shah and R.A. Malaney, Particle filters and position tracking in wi-fi networks, in: IEEE 63rd Vehicular Technology Conference, VTC, Vol. 2, 2006, pp. 613-617.

[59] Z. Shah, R.A. Malaney, X. Wei and K. Tai, Experimental deployment of particle filters in wifi networks, in: IEEE International Conference on Communications, ICC'07, 2007, pp. $4692-4697$.

[60] R. Singh, M. Guainazzo and C.S. Regazzoni, Location determination using wlan in conjunction with gps network (global 
positioning system), in: IEEE 59th Vehicular Technology Conference, VTC, Vol. 5, 2004, pp. 2695-2699.

[61] skyhookwireless, Skyhook ${ }^{\circ}$, 2013, URL http://www. skyhookwireless.com/, Detailed information can be found on the press site, see articles from 30/06/2008 (XPS), 09/02/2007 (SiRF), 30/09/2008 (Broadcom), 17/11/2008 (Qualcomm), 16/02/2009 (Texas Instruments), (visited on 15/02/2013).

[62] G. Sun, J. Chen, W. Guo and K.J.R. Liu, Signal processing techniques in network-aided positioning: A survey of stateof-the-art positioning designs, IEEE Signal Proc Mag 22(4) (2005), 12-23.

[63] S. Tekinay, E. Chao and R. Richton, Performance benchmarking for wireless location systems, IEEE Commun Mag 36(4) (1998), 72-76.

[64] D.H. Titterton and J.L. Weston, Strapdown Inertial Navigation Technology, 17th edn, Peter Peregrinus Ltd, 2004.

[65] A.W. Tsui, Y.-H. Chuang and H.-H. Chu, Unsupervised learning for solving rss hardware variance problem in wifi localization, Mobile Networks and Applications 14(5) (2009), 677691, URL http://dx.doi.org/10.1007/s11036-008-0139-0.

[66] S.J. Vaughan-Nichols, Will mobile computing's future be location, location, location? Computer 42(2) (2009), 14-17.

[67] T. Vaupel, J. Seitz, F. Kiefer, S. Haimerl and J. Thielecke, Wifi positioning: System considerations and device calibration, in: International Conference on Indoor Positioning and Indoor Navigation (IPIN), 2010.

[68] G.A. Vecchione, D. Palenzuela, M. Toledo, J.J.R. de Cáceres, D. Kubrak, Y. Capelle, G. Seco, J. Lopéz-Salcedo, P. Tiley, A. Consoli, D. Jiménez-Baños and G. Lopéz-Risueño, Dingpos, a gnss-based multi-sensor demonstrator for indoor navigation: Preliminary results, in: Proc. of IEEE/ION PLANS, 2010, pp. 113-121.

[69] M. Vossiek, L. Wiebking, P. Gulden, J. Wieghardt, C. Hoffmann and P. Heide, Wireless local positioning, IEEE Microw Mag 4(4) (2003), 77-86.

[70] X. Wang, A.K.-S. Wong and Y. Kong, Mobility tracking using gps, wi-fi and cell id, in: International Conference on Information Networking (ICOIN), 2012, pp. 171-176.

[71] Y.-H. Wang, Positioning with wireless local area networks and wlan-aided global positioning systems, 2004,
Pat. US2004162084 (A1), Atheros Communications, Inc. 19/08/2004.

[72] M. Weyn and F. Schrooyen, A wi-fi assisted gps positioning concept, in: Proc. of the European Conference on the Use of Modern Information and Communication Technologies, ECUMICT, 2008.

[73] L. Xu, S. Zhang, J. Quan and X. Lin, Vehicle positioning using wi-fi networks and gps/dr system, in: 5th International Conference on Mobile Ad-hoc and Sensor Networks, MSN'09, 2009, pp. 287-293.

[74] R. Yamasaki, A. Ogino, T. Tamaki, T. Uta, N. Matsuzawa and T. Kato, Tdoa location system for ieee $802.11 \mathrm{~b}$ wlan, in: IEEE Wireless Communications and Networking Conference, Vol. 4, 2005, pp. 2338-2343.

[75] M. Yan, X. Yubin and C. Xiuwan, Wireless local area network assisted gps in seamless positioning, in: International Conference on Computer Science and Electronics Engineering (ICCSEE), Vol. 3, 2012, pp. 612-615.

[76] F. Yang and D. Aoshuang, A solution of ubiquitous location based on gps and wi-fi ulgw, in: Ninth International Conference on Hybrid Intelligent Systems, HIS'09, Vol. 2, 2009, pp. $260-263$

[77] S.-C. Yeh, W.-H. Hsu, M.-Y. Su, C.-H. Chen and K.-H. Liu, A study on outdoor positioning technology using gps and wifi networks, in: International Conference Networking, Sensing and Control, ICNSC'09, 2009, pp. 597-601.

[78] S.-C. Yeh, W.-H. Hsu and Y.-S. Chiou, Adaptive-weighting schemes for location-based services over heterogeneous wireless networks, in: IEEE 71 st Vehicular Technology Conference (VTC 2010), 2010.

[79] P.A. Zandbergen, Accuracy of iphone locations: A comparison of assisted gps, wifi and cellular positioning, $T$ Gis 13 (2009), 5-25, URL http://dx.doi.org/10.1111/j.1467-9671. 2009.01152.x.

[80] S. Zirari, P. Canalda and F. Spies, Wifi gps based combined positioning algorithm, in: IEEE International Conference on Wireless Communications, Networking and Information Security (WCNIS), 2010, pp. 684-688. 